\title{
The interrelationship between emotion, cognition, and bilingualism
}

\author{
Crystal J. Robinson and Jeanette Altarriba* \\ University at Albany, State University of New York \\ *jaltarriba@albany.edu
}

\begin{abstract}
Research on the representation of emotion in human memory has focused on the ways in which words that label an emotion (e.g., love, joy) or represent emotional components (e.g., death, butterfly) are learned, stored, and retrieved from memory. The current work reviews the ways in which these types of words have been distinguished from concrete and abstract words, the types of methodologies used to distinguish among word groups, and the ways in which these words are automatically processed in the bilingual speaker. While emotion words may be more readily processed and retrieved when they appear in the first language, other word types that are neutral with regards to arousal and valence may be processed similarly across languages. The current work also illustrates the ways in which this knowledge is important in advancing theories of language and cognition, attention, perception, and mental health. Future directions are discussed that elucidate the further applications of these interesting lines of research.
\end{abstract}

Keywords: emotional language; codeswitching; emotion word processing; bilingualism and emotion; bilingualism.

\section{Introduction: Why study emotion?}

The ways in which we process and interact with emotional stimuli have received increased attention in the fields of psychology and linguistics (see e.g. Altarriba 2013). Emotion is a pervasive aspect of how we interact with the world around us. Furthermore, it is becoming apparent that the processing of emotion may depend upon the cultural and linguistic environment of the individual (e.g., Kim and Starks 2008; Wang 2011). In the current paper, an emphasis will be placed on the specific emotion terms that speakers use, and how this usage may interact with certain cognitive and linguistic factors. Specifically, we will discuss how using emotional language can interact with cognition when an individual is working within two languages (i.e., is a bilingual speaker). 
Research on emotion word usage across languages indicates that the set of emotion terms available in any given language is unique and reflective of the cultural context surrounding the language. These differences are often representative of a culture's perspective on people's ways of feeling (de Mendoza 2008). Furthermore, languages also differ in the relative size and character of their affective lexicon, such that the number of words available to describe emotions can differ greatly between languages. For example, in English there are well above 100 words in the affective lexicon, whereas in the Malaysian language, Chepang, there are less than 10 words in the affective lexicon (Altarriba 2003). The number of available emotion terms in a speaker's language has also been shown to correlate with emotional intelligence for that speaker. This is because the ability to recognize, name, and understand human emotions has been linked to a lower incidence of depression in both children and adults, overcoming of eating disorders, as well as an overall reduction in mental illness (Goleman 1995).

Differences between language speakers could be in part due to differences in emotion knowledge exhibited by members of a specific culture. Emotion knowledge is thought to be culturally construed and formed through participation in everyday sociocultural practices. Individual-focused cultures (e.g., Western European cultures) tend to consider emotion a direct expression of the self. In community-focused cultures (e.g., East Asian cultures) there is a premium placed on social harmony and group interests, and emotion is seen as destructive to this harmony in many cases. As a result, when asked to judge the emotional nature of a story or describe situations that likely provoke emotions, members of individual-focused cultures outperformed members of community-focused cultures. Furthermore, this increase in emotion knowledge is thought to reflect an increase in the ability for members of individual-focuses cultures to access earlier and more detailed accounts of childhood memories, as these memories are often bound up in their emotional context (Wang 2011).

Taken together, this evidence suggests that the use and processing of emotion across languages plays a pervasive role in everyday cognitive processing that carries over into a number of applied fields. These fields include health and wellness counseling, eyewitness testimony, and interpersonal relationships (Altarriba and Morier 2004; Altarriba and Santiago-Rivera 1994). This paper will go into specific detail concerning how bilinguals process emotion relative to monolinguals and how these differences affect the applied usage of emotion across a bilingual's first and second language. 


\section{Emotion word representation}

Before beginning with the specifics of how emotion words are processed, it is necessary to clearly define what is meant by an emotion word. Three basic distinctions need to be made. First, there is the distinction between concrete and abstract words. Concrete words describe real-world physical objects things, people, and creatures - for which you have a distinct mental image for e.g. "bed", "dog", and "flower." Abstract words, on the other hand, such as "freedom" refer to ideas, beliefs, or mental notions and have no easily referable mental image. Second, it was originally suggested that emotion words could be placed under the larger umbrella of abstract words. However, evidence suggests that emotion words are in fact not processed in the same way as abstract words (Altarriba et al. 1999), calling for a different distinction, in which emotion words can be considered in their own class. Lastly, recent work has revealed that emotion words need to be examined separately from what are referred to as emotion-laden words (Altarriba and Bauer 2004; Altarriba and Canary 2004). This dichotomy defines an emotion word as one that specifically refers to a given feeling (e.g., love, hate, anger, joy, etc.) and an emotion-laden word as one that refers to a thing or event that may evoke a given feeling (e.g., gifts, blood, war, etc.). Hence, after making this third distinction, we are left with four classes of words, concrete, abstract, emotion, and emotion-laden. The following sections will demonstrate the ways in which each of these categories differs with regards to cognitive processing, specifically, memory and attention.

The differentiation between abstract and emotion words came about due to growing evidence that words with emotional content were not behaving in the same manner as abstract words in typical processing tasks. It is the case that abstract words have a processing disadvantage relative to concrete words. Specifically concrete words tend to be recalled and recognized better than abstract words (Paivio 1971, 1986; Shwanenflugel et al. 1988). However, subsequent research revealed that emotion words were classified in a different fashion from both abstract and concrete words with regards to imagability and context availability, or the ease with which participants can think of a particular event corresponding to the given word (Altarriba et al. 1999). Furthermore, in recall tasks emotion words, both negative and positive, were found to have a processing advantage relative to both abstract and concrete words. Specifically, people tend to have better memory for emotion words than both abstract and concrete words (Altarriba and Bauer 2004). It is im- 
portant to note that the previous two studies did not make the distinction between emotion and emotion-laden processing. Further research into processing differences among word types would reveal this distinction. However, this research does reveals that emotion words play a specific, and perhaps, independent role in language processing, particularly with regards to memory.

To examine the role that emotion plays in attention, tasks that directly measure attentional mechanisms, such as the dot-probe task, have been employed (Sutton and Altarriba 2011). In the dot-probe task, participants are presented with a negative emotion (e.g., fear), positive emotion (e.g., happiness), or emotionally neutral word (e.g., seat) on one side of the computer screen or the other. Once the word disappears, a dot appears either on the same side or opposite side as the word. The participant's task is to simply indicate which side of the screen the dot is on. In this paradigm, participants are slower at making their judgment when the dot appears on the side opposite of where a negative emotion word appeared and faster when the dot appears on the same side as a negative emotion word. These effects gradually dissipate in the positive and neutral conditions, suggesting that our attention is automatically drawn to the negative words, and thus it is more effortful to draw our attention away, and focus on the location of the dot.

Following the notion that emotion words represent their own category within our linguistic system, researchers began to question whether all emotion words are processed similarly. Perhaps it is the case that the category of emotion words should be broken down even further. Affective priming studies have investigated this assumption by measuring reaction times for making a lexical decision. In the typical paradigm, participants are presented with an emotion, emotion-laden, or neutral word, and then presented with a letter string. The task is to determine whether the letter string is a word or a nonword as quickly and as accurately as possible. An example of this procedure is depicted in Figure 1. Emotion words, particularly negative emotion words, typically interfere with the lexical decision task, resulting in a longer reaction time. Furthermore, in this paradigm, it is typical for emotion words to interfere to a greater degree than emotion-laden words, making the case that emotion and emotion-laden words are not represented or processed in the same fashion (Altarriba and Bauer 2004). 


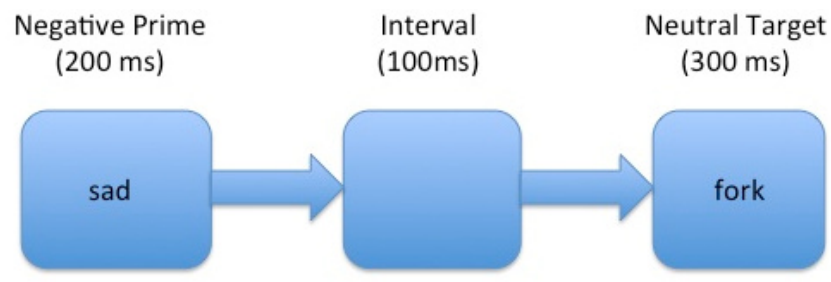

Figure 1. Lexical decision task procedure.

\section{Emotion word representation in bilingual speakers}

Examining emotion word processing from the bilingual perspective allows for an examination of the extent to which varying word types are processed the same across two languages. It can be the case that meaning representation of a particular word in a bilingual's first language can completely overlap, partially overlap, or exhibit no similarities with meaning representations of that same word in a bilingual's second language (de Groot 1992). Examination of differences in emotion word processing have led to insight as to whether cultural factors, linguistic factors, or a dynamic interaction between the two are playing a key role in cognitive processing (Altarriba 2003). The key question when examining emotion word processing in the bilingual speaker is whether or not emotion and emotion-laden words behave similarly across both languages for a bilingual.

A critical assumption for the study of emotion word processing in bilinguals is that emotion can be activated across both your first and second language. Furthermore, it is assumed that the representation of emotion across languages is more or less equivalent, such that emotion words enhance recall and interference across all languages. With regards to the representation of emotion, various studies examining the way bilinguals remember words with emotional content support this assumption. It has been found that bilinguals tend to remember emotion and emotion-laden words better than neutral words across both the first and second language. Furthermore, taboo words with emotional content tend to produce the best memory. This has been seen across the bilingual's first and second languages. Specifically, highly proficient Turkish-English bilinguals exhibited enhanced recall for emotion words across both Turkish and English (Ayçiçeği-Dinn and Caldwell-Harris 2009). 
A similar paradigm has been examined across early Spanish-Catalan and late Spanish-English bilinguals (Ferré et al. 2010). The authors did in fact find evidence in support of similar emotion representation across varying levels of proficiency. These studies do suggest that emotion words are processed in a similar fashion across a bilingual's first and second language, such that they enhance memory across both the first and second language. However, it is important to note that some studies have found evidence that a significant emotion effect either does not occur in a bilingual's second language or only occurs in a second language when the speaker is highly proficient in that language (Degner et al. 2011). Furthermore, this evidence only suggests that an emotion effect can occur across both of a bilingual's languages, making no suggestions concerning the degree to which emotion is activated across the first and second language for the bilingual speaker.

The automatic activation of emotion has been examined from the perspective of attentional mechanisms. The Emotion Stroop task (see Figure 2) provides evidence that when attention is involved, emotion does affect processing across a bilingual's first and second language. In the Emotion Stroop task, as is the case with English monolinguals, when the words presented are emotion words (e.g., love, hate, fear, etc.), participants tend to exhibit slower reaction times for naming the provided ink color. This effect has been shown across a bilingual's first and second language (Sutton et al. 2007), and suggests that emotion words are automatically drawing the participant's attention away from the target task (naming the ink color). Eilola et al. (2007) found that Finish-English bilinguals exhibited emotion interference across both the first and second language, but only when these bilingual speakers were highly proficient in both Finnish and English. These results further demonstrate how important it is to consider the bilingual speaker's proficiency in each of their two languages. It is important to note that the two previous studies did not compare differences between emotion and emotion-laden word processing. This distinction will be discussed further in the following two paragraphs.

To further examine the automatic activation of emotion, the Affective Simon Task was administered to English-speaking monolinguals and Spanish-English bilinguals. In the Simon Task, participants are asked to classify words in white based upon whether they are positive or negative. Conversely, words written in blue or green are to be classified on the basis of color. Words were either emotion words (e.g., happy, anxious) or emotion-laden words (e.g., dream, shark). Both negative and positive emotion words 


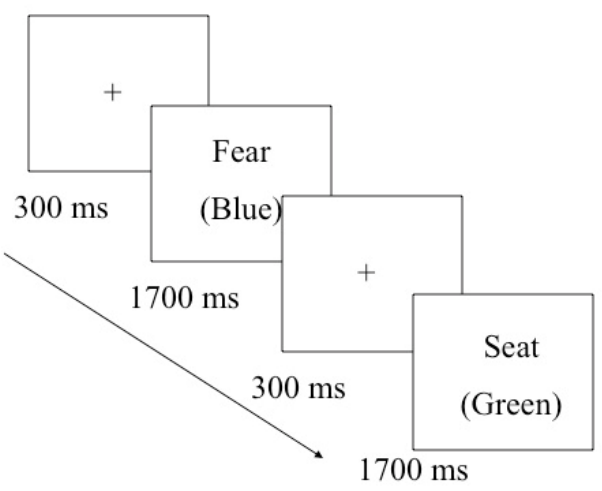

Figure 2. Emotion Stroop task.

produced a Simon Effect, insomuch as the emotionality of words not written in white was automatically activated, causing color classification to take longer. This occurred for both monolinguals and bilinguals. For bilinguals, these effects persisted across their first and second language. These results provide further evidence that affective coding can occur across a bilingual's first and second language for bilinguals who are highly proficient in their second language (Altarriba \& Basnight-Brown 2011). Word processing effects exhibited between emotion and emotion-laden words are outlined in the following paragraph.

Many studies that have examined emotion representation and memory across languages have employed a vague definition of emotion, not distinguishing between emotion-laden words and emotion words described earlier. In one study, Spanish-English bilinguals were asked to rate words based upon their concreteness, imagability, and context availability. Participants were shown words in both Spanish and English. Altarriba (2003) found that emotion and emotion-laden words differed with regards to concreteness, imagability, and context availability, suggesting differences in their representations. Spanish and English words were given similar ratings across participants. These results lend support to the claim that emotional representations can be activated across both of a bilingual's languages. Expanding upon this differentiation, Altarriba and Basnight-Brown (2011) found that the interference caused by negative emotion words was twice that of negative emotion-laden words. Results such as these demonstrate the necessity of considering emo- 
tion and emotion-laden words as two separate categories. Although the previously mentioned studies demonstrate that emotion and emotion-laden processing effects are exhibited across a bilingual's first and second language, another question of interest is whether the emotion effect is as strong in the second language as it is in the first.

To conclude the current section, a popular theme in the literature on bilingualism is that emotions are stronger if experienced in the native language. Many studies aim to determine whether evidence of the phenomenon could be provided by measures of arousal when processing words with emotional content (Eilola and Havelka 2011; Simcox et al. 2012). In one study, skin conductance levels of native and non-native English speakers were measured during emotional and taboo Stroop tasks. Although, as mentioned earlier, there are rarely any differences in performance on this task between the first and second language, the skin conductance measurement did reveal differences between the native and non-native participants. Native English speakers responded with significantly higher anxiety to negative and taboo words when compared with neutral and positive words. This difference was not observed in non-native speakers (Eilola and Havelka 2011). Thus, the level of arousal produced by the negative and taboo words is greater in one's native language. In another study, Spanish-English bilinguals who were more proficient in their second language (English) were asked to read aloud taboo and neutral words in either language while skin conductance measurement served as the measure of arousal. Overall, the bilingual's first language (Spanish) yielded greater arousal and was processed less efficiently than the second. This suggests that processing in the first language was more effortful for participants. Critically, arousal and processing efficiency were greater for taboo words than for neutral words presented in the second language but not the first. These results suggest that frequent use and proficiency of a language can impact whether or not emotional content will lead to increased arousal (Simcox et al. 2012). This particular study indicates that it is necessary to consider both language dominance and proficiency as important variables when examining emotion processing and use from the bilingual perspective.

In summary, it is clear that emotion can be activated across both of a bilingual's languages. This effect is likely moderated by the speaker's proficiency level within each of the two languages. Specifically, when a bilingual is highly proficient in both their first and second language, emotional activation tends to be equivalent in both languages (Altarriba and Basnight-Brown 2011; Ayçiçegi-Dinn and Caldwell-Harris 2009; Eilola et al. 2007; Ferré et 
al. 2010; Sutton et al. 2007). However, if the bilingual speaker is not equally proficient in each of his or her languages, it is likely that emotion will be activated more strongly in the most proficient of the two languages (Degner et al. 2011). Lastly, although behavioral measures, such as memory and attention tasks show that emotion can be equally activated across both of a bilingual's languages, physiological evidence suggests that negative emotion words result in greater arousal when presented in the first language, as long as the bilingual's second language is not the dominant language of use (Eilola and Havelka 2011; Simcox et al. 2012). This last point, as will be discussed in the following sections, is of particular importance when considering how bilinguals use language when describing emotionally distressing events.

\section{Emotion and language use}

The way in which emotion is processed for the bilingual speaker ultimately affects the patterns of language use exhibited by the bilingual. These patterns are reflective of findings from within the bilingual emotion word processing literature. Qualitative examination of bilingual language use reveals that bilingual speakers tend to have language specific preferences when expressing emotions, such as anger and love, or even religious sentiment. Particularly, there is often a language that a bilingual prefers to pray in. This is most likely the language in which the speaker predominantly expressed religious sentiment in the past (Bloom and Beckwith 1989). Further examination has found that a bilingual's first language is often the language of emotional expressiveness of various kinds (Dewaele and Nakano 2012). However, when the emotional content, particularly of past events or memories, is too anxiety provoking a bilingual is likely to prefer his or her second language when discussing such events or memories. This behavior allows for the bilinguals to distance themselves from negative events and feelings (Dewaele and Pavlenko 2002). It is important to note that although much of the qualitative work has been concerned with bilingual language use in terms of a native language versus non-native language dichotomy, language dominance patterns may also be playing an important role in language use patterns, particularly with regards to emotion. Recent work has shown that as a bilingual begins to lose language abilities in his or her first language, often through becoming more dominant in the second language, they also begin to express emotions in their 
second language with more fluency than in their first (Kim and Starks 2008). This research requires one to consider both the native language of the speaker, as well as the speaker's dominant language of use.

The fact that the bilingual may prefer to speak one language rather than the other in a given situation can cause the speaker to switch from one language to another. Thus, if the situation or topic of conversation changes, the language of use has the potential to change as well. A bilingual's ability to mix their two languages during communication can lead to telling insight regarding how bilinguals are processing emotion words in both their first and second language. This ability is referred to as code switching. Code switching occurs when bilinguals substitute a word or phrase from one language with a word or phrase from another language. Code switching can serve as an emotional distancing function, allowing the speaker to feel more free to discuss emotional or anxiety arousing topics (Altarriba and Heredia 2001; Wu and Thierry 2012). As was previously mentioned, this is likely due to the fact that obscene and emotionally charged words generate less anxiety in the second or non-dominant language. As will be noted in the following section, code switching may in fact be beneficial for the bilingual in many applied settings that require the speaker to bring up emotionally charged events and memories. Furthermore, when a bilingual does switch between languages, whether by choice or out of necessity, he or she does report perceptions of feeling "different" in the second language. Specifically, speakers report feeling less logical and emotional and increasingly fake when switching from their first to their second language (Dewaele and Nakano 2012). The following section will demonstrate that understanding how and in what context bilinguals express emotion in a given language can be useful in various applied settings.

\section{Applied issues in emotion processing}

Bilingualism affects memory in a pervasive fashion, such that memories for events are dependent upon a specific language as a retrieval cue. Specific information may not be available in both languages. Javier et al. (1993) examined memory for personal events in Spanish-English bilinguals. Participants were asked to describe an interesting or dramatic event from personal history for five minutes in their native language. They were then asked to retell the event in L2. Javier et al. found that the quality of description was richer in 
L1, and memories encoded in a specific language were better remembered in that same language, regardless of whether it was the native language or not. It is easy to see how this research is applicable to therapeutic settings. Schrauf (2000) found similar results when memories from childhood were examined. He found that memories experienced in the mother tongue were typically richer in terms of emotional significance, particularly when recalled in L1. Benefits of having two languages available for use during therapy are numerous, but include allowing the client to be more expressive. On the other hand, the second language can be used as a distancing function when recalling particularly painful life experiences since most emotion information is encoded in L1. Marcos (1976) defined this effect as the detachment effect, noting the tendency for L2 to be devoid of emotion. This occurrence may be due to the cultural or emotional context in which each of the two languages is learned (Altarriba 2003). Typically, L2 is learned in a formalized and systematic environment, and speakers rarely have experiences with emotional conflict encoded in this language.

Specific findings in the area of counseling and mental health treatment mirror the theoretical findings discussed above. Altarriba and Santiago-Rivera (1994) examined the impact of using a bilingual's dominant language in a therapeutic setting. The benefit of this approach is that the client can use a broad vocabulary, allowing for language-specific information, such as emotional content, to be easily communicated. When these emotional events are discussed in the native language, they do tend to be more spontaneous, as the recall of repressed memories often occurs in the native language. In such cases, the use of the native language may also inhibit the client from discussing painful events, as emotional content may be too intense in one's first language. The use of the bilingual's second language in therapeutic settings may allow for the client to express emotionally charged events with a degree of distance, lessening the psychological strain of the situation. On the other hand, this discussion may be forced and void of particular details that may only present themselves in the first language. Furthermore, discussion in the second or non-dominant language may be difficult for the client if he or she does not possess the necessary vocabulary. Yet, if the non-native language is used in conjunction with the native language, the client can freely discuss emotional events while still accessing their dominant language vocabulary. Language mixing within a given therapeutic session requires the presence of a bilingual therapist. In such a scenario, a bilingual's unique abilities can be considered a benefit rather than a deficit (Santiago-Rivera and Altarriba 2002). 
The bilingual therapist often holds the role of guiding language use patterns within a particular session. Thus, it is necessary to examine the therapist's impact on the emotional language use of bilingual clients. To assess the extent to which bilingual therapists use both Spanish and English in therapy as a strategic tool, and to determine when and how bilingual clients switch from one language to another, Santiago-Rivera et al. (2009) examined the therapeutic styles, techniques, and client interactions of nine different bilingual therapists. Similar to previous studies, they found that bilingual clients would often discuss regular emotions in the second language, but when spontaneous emotional outbursts occurred, they most often occurred in the first language. Specifically, some therapists noted that clients would deal with regular anger emotions in English but when they explode it would be in Spanish (Santiago-Rivera et al. 2009).

The therapist would also use both languages, switching between the two to serve a particular purpose. Occasionally, the switch would occur to accentuate the commonalities between the client and therapist, or to simply form a bond. Some therapists also noted that the use of the client's native language was helpful in promoting disclosure of pertinent information. Therapists mentioned that using a client's native language demonstrated that they were understanding and reaching out to the clients, especially if they use a phrase or expression in Spanish from the community (Santiago-Rivera et al. 2009). The use of native sayings was also a helpful tool in increasing understanding between the therapist and client. Lastly, the use of two languages adds to the clarity of the therapeutic session, providing a benefit for both the therapist and the client.

In summary, bilingual clients switch to their native language spontaneously when recounting conflict based experiences and discussing very early memories. Interestingly, clients do tend to present themselves differently depending upon the language being spoken. On the therapist's end, the use of the client's native language is helpful in emphasizing commonalities, establishing credibility and trust, promoting disclosure, and drawing out emotions. These findings specifically underscore the importance of understanding how a bilingual speaker processes and uses emotional content. This understanding has an obvious impact on the effectiveness of therapy for the bilingual client, but is also necessary for understanding how the bilingual speaker communicates in various health and legal settings. Any scenario that requires the bilingual to call on emotional memories or express current emotions will benefit from such an understanding. 


\section{Conclusions}

The study of emotion word processing can yield important findings that have relevance to situations ranging from the learning of new languages, and interpreting and translating between languages, to understanding the dynamics involved in developing and maintaining mental health and well-being. The current paper explored the ways in which emotion word representation and processing occur in human memory, the interaction between emotion and cognition in communication and a variety of cognitive, perceptual, and memory tasks, and the contributions of emotion research to the applied areas of counseling and therapy. Future research directions should delve into the representation of words, pictures, and images that range from positive to neutral to negative. Also, it is important to vary levels of arousal in order to consider how the intensity of a given emotion may affect processing. Clearly, emotion affects key components of behavior that include motivation, selfcontrol, and self-regulation - areas of research that have yet to uncover the underlying mechanisms that serve to promote and maintain the human emotional system.

\section{References}

Altarriba, J. 2003. "Does cariño equal "liking"? A theoretical approach to conceptual nonequivalence between languages". International Journal of Bilingualism 7(3). 305-322.

Altarriba, J. 2013. "Emotion, memory, and bilingualism". In: Altarriba, J. and R. Heredia (eds.), Foundations of bilingual memory. New York, NY: Springer. 85203.

Altarriba, J. and D.M. Basnight-Brown. 2011. "The representation of emotion vs. emotion-laden words in English and Spanish in the Affective Simon Task". International Journal of Bilingualism 15(3). 310-328.

Altarriba, J. and L.M. Bauer. 2004. "The distinctiveness of emotion concepts: A comparison between emotion, abstract, and concrete words". The American Journal of Psychology. 389-410.

Altarriba, J., L.M. Bauer and C. Benvenuto. 1999. "Concreteness, context availability, and imagability ratings and word associations for abstract, concrete, and emotion words". Behavior Research Methods, Instruments, \& Computers 31(4). 578-602.

Altarriba, J. and T.M. Canary. 2004. "The influence of emotional arousal on affective priming in monolingual and bilingual speakers". Journal of Multilingual and Multicultural Development 25(2-3). 248-265. 
Altarriba, J. and R. Heredia. 2001. "Bilingual language mixing: Why do bilinguals code-switch?" Current Directions in Psychological Science 10(5). 164-168.

Altarriba, J. and R.G. Morier. 2004. "Bilingualism: Language, emotion and mental health". In: Bhatia, T.K. and W.C. Rithcie (eds.), The handbook of bilingualism. Oxford: Blackwell. 250-280.

Altarriba, J. and A.L. Santiago-Rivera. 1994. "Current perspectives on using linguistic and cultural factors in counseling the Hispanic client". Professional Psychology: Research and Practice 25(4). 388-397.

Ayçiçegi-Dinn, A. and C.L. Caldwell-Harris. 2009. "Emotion-memory effects in bilingual speakers: A levels-of-processing approach". Bilingualism: Language and Cognition 12(3). 291-303.

Bloom, L. and R. Beckwith. 1989. "Talking with feeling: Integrating affective and linguistic expression in early language development". Cognition \& Emotion 3(4). 313-342.

de Groot, A.M. 1992. "Determinants of word translation". Journal of Experimental Psychology: Learning, Memory and Language 18. 1001-1018.

de Mendoza, H.A. 2008. "The problem of translation in cross-cultural research on emotion concepts (Commentary on Choi \& Han)". International Journal for Dialogical Science 3(1). 241-248.

Degner, J., C. Doycheva and D. Wentura. 2011. "It matters how much you talk: On the automaticity of affective connotations of first and second language words". Bilingualism: Language and Cognition 15(1). 181-189.

Dewaele, J.-M. and S. Nakano. 2012. "Multilinguals' perceptions of feeling different when switching languages". Journal of Multilingual and Multicultural Development 34(2). 107-120.

Dewaele, J.-M. and A. Pavlenko. 2002. "Emotion vocabulary in interlanguage". Language Learning 52(2). 263-322.

Eilola, T.M. and J. Havelka. 2011. "Behavioural and physiological responses to the emotional and taboo Stroop tasks in native and non-native speakers of English". International Journal of Bilingualism 15(3). 353-369.

Eilola, T.M., J. Havelka and D. Sharma. 2007. "Emotional activation in the first and second language". Cognition and Emotion 21(5). 1064-1076.

Ferré, P., T. García, I. Fraga, R. Sánchez-Casas and M. Molero. 2010. "Memory for emotional words in bilinguals: Do words have the same emotional intensity in the first and in the second language?" Cognition and Emotion 24(5). 760-785.

Goleman, D. 1995. Emotional intelligence. New York, NY: Bantam.

Javier, R.A., F. Barroso and M.A. Muñoz. 1993. "Autobiographical memory in bilinguals". Journal of Psycholinguistic Research 22. 319-338.

Kim, S.H.O. and D. Starks. 2008. "The role of emotions in L1 attrition: The case of Korean-English late bilinguals in New Zealand". International Journal of Bilingualism 12(4). 303-319.

Marcos, L.R. 1976. "Bilinguals in psychotherapy: Language as an emotional barrier". American Journal of Psychotherapy 30(4). 552-560

Paivio, A. 1971. Imagery and verbal processes. New York, NY: Holt, Rinheart \& Winston. 
Paivio, A. 1986. Mental representation: A dual-coding approach. New York, NY: Oxford University Press.

Santiago-Rivera, A.L. and J. Altarriba. 2002. "The role of language in therapy with the Spanish-English bilingual client". Professional Psychology: Research and Practice 33(1). 30-38.

Santiago-Rivera, A.L., J. Altarriba, N. Poll, N. Gonzalez-Miller and C. Cragun. 2009. "Therapists' views on working with bilingual Spanish-English speaking clients: A qualitative investigation". Professional Psychology: Research and Practice 40(5). 436-443.

Schrauf, R.W. 2000. "Bilingual autobiographical memory: Experimental studies and clinical cases". Culture \& Psychology 6(4). 387-417.

Schwanenflugel, P.J., K.K. Harnishfeger and R.W. Stowe. 1988. "Context availability and lexical decisions for abstract and concrete words". Journal of Memory and Language 27(5). 499-520.

Simcox, T., M. Pilotti, S. Mahamane and E. Romero. 2012. "Does the language in which aversive stimuli are presented affect their processing?" International Journal of Bilingualism 16(4). 419-427.

Sutton, T.M. and J. Altarriba. 2011. "The automatic activation and perception of emotion in word processing: Evidence from a modified dot probe paradigm". Journal of Cognitive Psychology 23(6). 736-747.

Sutton, T.M., J. Altarriba, J.L. Gianico and D.M. Basnight-Brown. 2007. "The automatic access of emotion: Emotional Stroop effects in Spanish-English bilingual speakers". Cognition and Emotion 21(5). 1077-1090.

Wang, Q. 2011. "Autobiographical memory and culture”. Online readings in psychology and culture, Unit 5.

(Retrieved from http://scholarworks.gvsu.edu/orpc/vol5/iss2/2.)

$\mathrm{Wu}$, Y.J. and G. Thierry. 2012. "How reading in a second language protects your heart". The Journal of Neuroscience 32(19). 6485-6489.

\author{
Address for correspondence \\ Jeanette Altarriba \\ Department of Psychology \\ Social Science 399 \\ University at Albany, State University of New York \\ 1400 Washington Avenue \\ Albany, New York 12222 \\ USA \\ jaltarriba@albany.edu
}

